\title{
Expect the unexpected: memorable experiences at the 2018 Council of Science Editors annual meeting
}

\author{
Cheol-Heui Yun \\ Department of Agricultural Biotechnology, Seoul National University, Seoul, Korea
}

The 2018 Council of Science Editors (CSE) annual meeting was held at the Hilton New Orleans Riverside, New Orleans, Louisiana on May 5-8, 2018. It consisted of 2 days of short courses (May 5 and 6) and 2 days of the main conference (May 7 and 8). The hotel where I stayed was the same location as the meeting venue, and it provided a unique view, as it was positioned against the banks of the Mississippi, so that one could watch the ships come and go.

At the meeting, I had the chance to closely engage with not only its overall program, including its content, speakers, and interactions between speakers (for oral and poster presentations) and the audience, but also subjects of my own interest-namely, ethical issues and related discussions. Moreover, as is always true at scientific meetings, it was a unique opportunity to meet and speak with organizers, speakers, poster presenters, and vendors. Indeed, it was extremely nice to see Patricia Baskin (immediate past president of the CSE) and Amy McPearson (director of publications for the Botanical Society of America and managing editor of the American Journal of Botany, 2018-2021), who visited the Korean Council of Science Editors in Korea in 2017 for the lecture on ethical issues. Seeing them was a pleasant surprise to me, since they were the only attendees and organizers I knew upon arrival; however, they were kind enough to introduce me to many of the people associated with the meeting.

The CSE short courses, composed of 5 parts (short course for journal editors, for publication management, on journal metrics, on publication ethics, and for manuscript editors), were held during the preconference before the CSE annual meeting. The objective of the short course on publication ethics was to introduce, address, and review the ethical issues outlined in the 'CSE white paper on promoting integrity in scientific journal publications' for those involved in the publication process, such as managing editors, publication managers, and journal staff. In this report, I would like to primarily focus on the short courses since only limited information about these courses is available on the conference website, whereas the main themes for previous years can easily be found (https://www.councilscienceeditors.org/events/previousannual-meetings/cse-2018-annual-meeting/). The course covered a range of ethical issues, from basic to specific, for newcomers and editors or journal staff members using the 'CSE white paper on publication ethics' as a guide. It also dealt with conflicts of interest, duplicate publications, piracy and plagiarism, data misrepresentation, image fraud, authorship disputes, 
editorial independence, research misconduct, and preprint issues. A particularly interesting talk addressed how research transparency should be carefully cultivated by promoting reproducible results and being vigilant for statistical errors and tainted research, as wrongdoing in research leads to ethical issues including fraudulent authorship, fraudulent data, and eventually fraudulent publications. Additionally, a brief but interesting discussion was held about the policy of 'forced' open access in Germany, wherein the government has launched an initiative to provide funding for the publication of all German research.

Christine Cassy gave a talk entitled 'Journal editor 101: a survival kit' and she recommended the following steps for new journal editors: (1) reading through the most recent year of the journal (and, importantly, its competitors); (2) knowing the history and mission of the journal; (3) reviewing the budget and the business model, and asking staff members about these issues and carefully listening to their responses; (4) asking for a standard operating procedure or work flow; (5) familiarizing oneself with the editorial policy; (6) putting benchmarks in place; (7) carrying out a mock-up of the author experience through a real or realistic process; (8) choosing to make changes immediately or to wait 6 months based on a careful consideration of one's role (or the journal's role); and (9) reflecting and writing about one's entrance and exit, in a process of thinking forward and onward.

Patricia Baskin (the immediate past president of CSE and executive editor of Neurology journals, American Academy of Neurology) discussed how the journals of the American Academy of Neurology succeeded by becoming 'niche' journals and presented the following reasons and timing for pursuing such a strategy: (1) when a journal is at the stage of rejecting high quality articles; (2) if the open access space for a given field (i.e., Neurology: Neuroimmunology \& Neuroinflammation) may receive more attention; (3) to provide authors and readers what they want; and (4) to add value constantly, as assessed using market analyses and publishing metrics. She also explained that the following aspects of a journal are useful for recruiting manuscripts: a suitable concept of the journal, impact factor, listing in PubMed Central and Medline, quick processing times, rapid and continuous publication, and a strong editor and/or editorial board. Ways to recruit content include calls for papers from the publisher, press releases and ads, mailings to members and departments, inviting researchers from meeting abstracts (annual meetings, international meeting, poster sessions, presentations, preprint servers), and social media promotions.

Denis G. Baskin gave a talk entitled 'Using metrics to improve your journal' based on author-level, article-level, and journal-level metrics. There was a spirited discussion after the talk about how the impact factor affects editors and their performance, and as well as issues involving the Declaration on Research Assessment (DORA, https://sfdora.org) in relation to diversity and alternatives in journal metrics (Eigenfactor, SCImago Journal Rank, and Altmetric) and author-level indices (e.g., h-index, Kardashian index, and Google Scholar h5index) as follows:

DORA

On December 16, 2012, a group of editors and publishers of scholarly journals met at the Annual Meeting of the American Society for Cell Biology in San Francisco, CA, USA to discuss current issues related to how the quality of research performance is (and can be) evaluated and how the primary scientific literature is cited. This meeting led to the announcement of recommendations referred to as the San Francisco DORA, which intends to halt the practice of correlating a journal's impact factor to the merits of a specific scientist's contributions. It states that the impact factor should not to be used as a substitute "measure of the quality of individual research articles, or in hiring, promotion, or funding decisions". Furthermore, according to the DORA statement, this practice creates biases and inaccuracies in the appraisal of scientific research.

The second day was also exciting, and started with a session led by Elizabeth Blalock entitled 'Ethics in the editorial office: tools and guidelines for daily use.' She began by reviewing the authorship requirements of the International Committee of Medical Journal Editors as shown below (http://www.icmje. org/recommendations/browse/roles-and-responsibilities/defining-the-role-of-authors-and-contributors.html):

Authorship criteria recommended by the ICMJE

Substantial contributions to the conception or design of the work; or the acquisition, analysis, or interpretation of data for the work; AND

Drafting the work or revising it critically for important intellectual content; AND

Final approval of the version to be published; AND

Agreement to be accountable for all aspects of the work in ensuring that questions related to the accuracy or integrity of any part of the work are appropriately investigated and resolved.

She emphasized the role of editorial staff in policy enforcement and as advisors to editors. Then, she briefly touched on various ethical areas, including research ethics in animal and human trials, plagiarism, how Retraction Watch looks for publication problems, and image manipulation [1].

Patricia K. Baskin then gave a talk entitled 'Clarifying ethics policies in the instructions to authors.' She pointed out that some journals may only accept submissions from authors who use an institutional e-mail address, or may ask the corresponding author to add an institutional e-mail as a part of peer re- 
view ethics. She further suggested that clinical data deposit should not be considered as publication. She emphasized that two-thirds of retractions are for research misconduct, and that the organizations that look for publication problems such as plagiarism include Retraction Watch, SciFraud, Science Fraud, and PubPeer. She also discussed some tough questions, such as how editors should handle anonymous claims and complaints. There are no obvious answers to this issue, and it must be dealt with on a case-by-case basis. However, she emphasized that journals should look into potential problems. It was also interesting that there were some concerns about the term 'retraction,' as it seemed to be a harsh word. During the questionand-answer session, this issue was further discussed, and a few new terms were proposed, such as 'honest mistake' or 'partial retraction. However, those terms do not refer to the same issue as an actual 'retraction.' It was useful to learn that when journals publish retractions, it is important to declare that the work is no longer reliable due to the authors' misconduct. The key issue here is that the retraction should be informative. Otherwise, Retraction Watch will point out that a retraction is not helpful. In this context, I was delighted to hear the proverb "An ounce of prevention is worth a pound of cure."

This issue was further discussed after a talk by Christina Bennett (the American Physiological Society) on 'Correcting the literature.' Bennett explained that the options for correcting the literature are errata, acknowledgments of honest errors (which are handled through retraction and replacement), retractions (for non-reproducible or pervasive errors, duplicates, etc.), and expressions of concern. It was helpful to learn that when an unintentional, minor, and correctable error is made, journals should publish a corrigendum or correction letter with an apology.

I was impressed by the talk and demonstration by Eric Pesanelli (the American Physiology Society), entitled 'Rules and tools for addressing image manipulation.' He started the talk by showing case studies on image manipulation, demonstrating that since proactive regulation of images started in 2010, the number of ethics cases jumped from 134 to 251. Figure manipulation accounted for the most cases, he said. He showed actual cases using software tools such as Acrobat, Photoshop, and PowerPoint. The workflow when manipulation is identified is to notify the author(s) first, and then the editor(s).
Then, an exercise session was conducted, asking whether various cases were potential misconduct or wrongdoing, as follows: (1) if the wording and data are the same (yes, meaning potential misconduct); (2) if a different language is used (unlikely to be misconduct, but permission is needed); (3) if an item is drawn from an institutional repository (no, meaning that it would be acceptable); (4) if data are published from a public repository (no); (5) if items are extracted from articles and books (they should be used only with permission due to copyright issues); (6) if a paper has been previously published on an author's blog (yes); (7) if the same exact wording is found in a paper but with completely different data (yes); (8) if the same exact wording is used in the Methods (rephrasing is recommended).

Collectively, although it was my first chance to attend an annual meeting of the CSE, I had a fantastic experience and was able to learn about recent advances in journal editing and publishing. Furthermore, I was glad to have the opportunity to communicate with other editors and editorial staff. Through conversations with colleagues, I came to understand the editorial process of large society journals in the United States. The next CSE annual meeting will be held at the Hyatt Regency in Columbus, Ohio, the United States from May 4 to 7, 2019. I would like to recommend that Korean and Asian editors should attend this meeting to broaden their horizons as scholarly editors.

\section{Conflict of Interest}

No potential conflict of interest relevant to this article was reported.

\section{Acknowledgments}

This work was supported by a travel grant from the Korean Council of Science Editors (2018).

\section{Reference}

1. Rossner M, Yamada KM. What's in a picture? The temptation of image manipulation. J Cell Biol 2004;166:11-5. https://doi.org/10.1083/jcb.200406019 